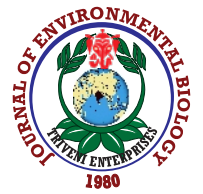

\title{
Isolation and characterization of bacteria from tomato and assessment of its plant growth- promoting traits in three economically important crops in Al-Ahsa region, Saudi Arabia
}

\author{
M.F. Aldayel' ${ }^{1}$ and A. Khalifa ${ }^{1,2 *}$ \\ ${ }^{1}$ Biological Sciences Department, College of Science, King Faisal University, Al-Ahsa-31982, Saudi Arabia \\ ${ }^{2}$ Botany and Microbiology Department, Faculty of Science, Beni-Suef University, Beni-Suef-62521, Egypt \\ *Corresponding Author Email : akhalifa@kfu.edu.sa
}

\section{Abstract}

Aim: This study aimed to isolate, characterize and assess the plant growth-promoting traits of bacterial isolates inhabiting the rhizosphere of Solanum lycopersicum L. (tomato) against three plants_Eruca sativa, Lepidium sativum and Raphanus sativum_from Saudi Arabia's Al-Ahsa region.

Methodology: A bacterial isolate designated as SLK10 was obtained from the rhizosphere of tomato grown in the Al-Ahsa region, Saudi Arabia. SLK10 was further characterized morphologically, biochemically and genotypically using $16 \mathrm{~S}$ rRNA gene sequencing. The roles in alleviating salt stress effects on three important economic crops were also assessed by implementing a cross-inoculation strategy.

Results: SLK10 formed a circular, non-pigmented and raised colony with an entire margin. The cells were rod shaped and Gram negative. SLK10 displayed multiple plant growth-stimulating features, such as the solubilization of inorganic phosphate and the production of phytohormones and acetoin. Comparative sequence analysis of $16 \mathrm{~S}$ rRNA gene revealed that SLK10 belonged to Pseudomonas monteilii, to which it exhibited $99.86 \%$ sequence homology. SLK10 significantly promoted the length of primary root, stem and number of leaves in Eruca sativa, Lepidium sativumand Raphanus sativus growing under $1000 \mathrm{ppm}$ salt stress. The number of leaves in $L$. sativum and $R$. sativus growing under 2000 ppm salt stress was substantially enhanced by SLK10.

Interpretation: SLK10 is a plant growth-promoting bacterium and can be used as a green fertilizer to increase soil fertility and plant productivity in the Eastern region of Saudi Arabia.

Key words: Pseudomonas species, Rhizobacteria, Rhizosphere, Salt stress, Solanum lycopersicum

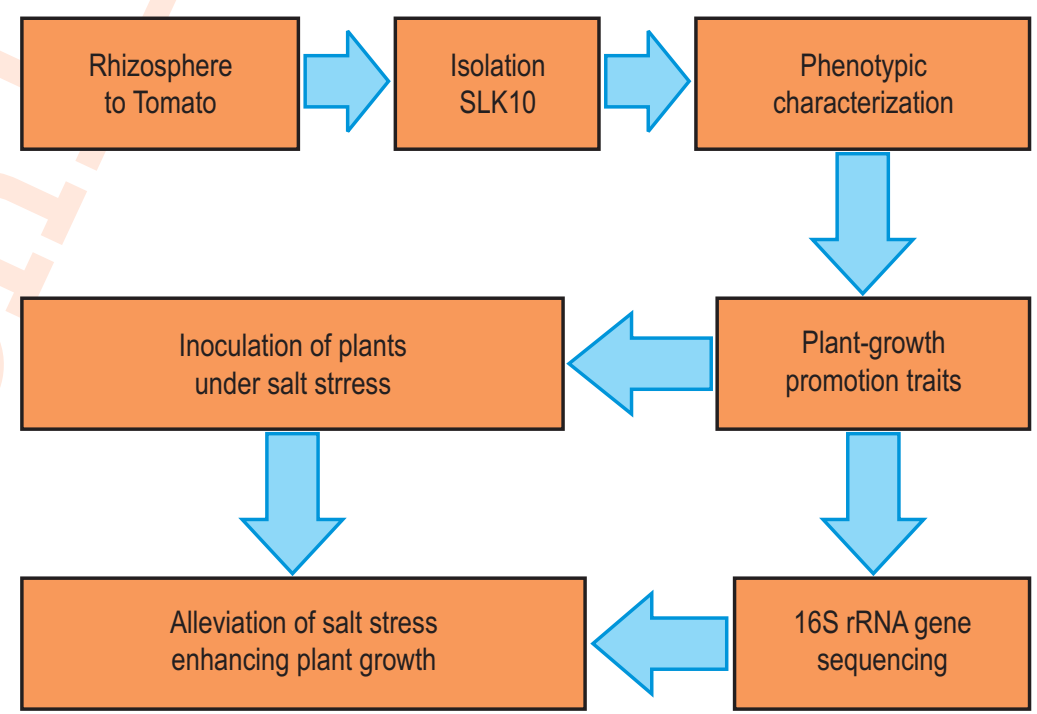

How to cite : Aldayel, M.F. and A. Khalifa: Isolation and characterization of bacteria from tomato and assessment of its plant growth-promoting traits in three economically important crops in Al-Ahsa region, SaudiArabia. J. Environ. Biol., 42, 973-981 (2021). 


\section{Introduction}

Abiotic stresses continue to adversely affect crop production and productivity. Damage from these stresses is responsible for significant economic losses worldwide. Salinity is a key environmental abiotic stress (Liao et al., 2020), and the increase in arable land salinization is likely to have worrying global consequences (Singh, 2021). Saudi Arabia's semi-arid and arid weather represents a substantial challenge to agriculture, and the issue of increasing salinity in cultivated land is becoming a prominent threat.

Salinity is a major obstacle limiting soil fertility and plant productivity, affecting a vast majority of the total area in Saudi. Elevated levels of salts exert negative effects on the morphological and physiological features of plants via multiple mechanisms, such as osmotic and oxidative stresses and ion toxicity; consequently, plant productivity is substantially decreased. The application of beneficial rhizobacteria, which inhabit the soil region adhered to plant roots, has become a promising strategy not only to alleviate salinity's harmful impacts but also to enhance plant growth. Rhizobacteria play their positive roles directly and indirectly (Shameer et al., 2018). Direct mechanisms include solubilization of inorganic phosphate, production of phytohormones and sequestration of iron. Nevertheless, the alleviation of abiotic stressors, such as salinity and drought, and the production of anti-phytopathogen compounds are among the indirect mechanisms via which rhizobacteria enhance plant growth (Shameer et al., 2018).

Numerous lines of evidence have documented that a wide array of different bacterial groups inhabit the rhizosphere of many plant species. Examples of such bacteria include Pseudomonas fluorescens, P. putida, Burkholderia phytofirmans and Microbacterium, all of which enhance the growth of different plant species such as wheat, rice and canola (Shameer et al., 2018). Furthermore, rhizobacteria exhibit a unique ability to ameliorate salinity constraints on plant hosts; for example, inoculation of Medicago polymorpha with Bacillus megaterium alleviated the negative effects of salinity stress (Chinnaswamy et al., 2018).

Pseudomonas is a bacterial genus that belongs taxonomically to the family Pseudomonadaceae within the class Gammaproteobacteria. Pseudomonas spp. are Gram-negative rods, inhabit various ecological niches and are medically and industrially important organisms. Furthermore, a wide metabolic versatility exists among members of this genus. Pseudomonas comprises 234 child taxa and is a widely published genus https://lpsn.dsmz.de/genus/Pseudomonas. Pseudomonas spp. also exist in the rhizosphere of various plant root like $P$. donghuensis in tomato (Ossowicki et al., 2017) and $P$. palleroniana in banana (Gamez et al., 2020) are examples of plant growth-promoting associations of Pseudomonas and plant species. P. monteilii was first obtained from human bronchial aspirate (Elomari et al., 1997); however, other strains from this species have been documented as plant growth-promoting rhizobacteria (Pandya and Desai, 2014).

Solanum lycopersicum L. (tomato) is a dicot plant that is grown for its edible fruits in many countries worldwide, including Saudi Arabia. Taxonomically, S. lycopersicum belongs to the Solanaceae family. It is the second most important vegetable crop in the world after potato. Tomato root system analysis has established the existence of a plant growth-promoting association with a wide array of PGPR, including Psudomonas sp. (Qessaoui et al., 2019), Chryseobacterium sp., Microbacterium sp., Rhizobium sp., Rhodococcus sp. and Agrobacterium sp. (Abbamondi et al., 2016). However, little is known about the growth-promoting rhizobacteria from tomato in the Al-Ahsa region of Saudi Arabia. Therefore, it is of interest to explore the plant growth rhizobacteria associated with the rhizosphere of tomato in Al-Ahsa and implement a crossinoculation strategy of three important plant species with potential candidates of associated rhizobacteria as eco-friendly biofertilizers.

Eruca sativa (rocket), Lepidium sativum (garden cress) and Raphanus sativus (radish) are three economically important herbaceous medicinal plant species that belong to the Brassicaceae family. Knowledge regarding these species developed centuries ago in eastern regions and then spread worldwide. Garden cress is recognized for its pungent odour due to the presence of several volatile oils and has been used to treat various conditions, such as respiratory disorders, muscle pain, inflammation and bone fractures (Raza et al., 2020). The leaves, seeds and aerial parts' extracts have been found to produce alkaloids, flavonoids, glycosides, polyketides, vitamins, minerals, proteins, fats and carbohydrates, all of which give the plant its hepatoprotective, antihypertensive, diuretics, fracture healing, respiratory disorder healing, antimicrobial, milk production, antiinflammation, antioxidant, laxative, chemoprotective and many other therapeutic properties (Raza et al., 2020). Additionally, raw rocket and radish are edible vegetables and provide a unique taste when added to salads and other dishes. Young plants are used as green fodder. Rocket is also used as a biodiesel or lubricant crop (Raza et al., 2020).

Despite numerous studies having documented the potential benefits of rhizobacteria in the Kingdom, including in AlAhsa (Khalifa and Almalki 2015; Khalifa et al., 2016; Khalifa et al., 2019, Khalifa et al., 2020), few studies have focused on the role of rhizobacteria in alleviating salinity stress in plants. Recently, Khalifa et al. (2019) reported that the bacterial species Bacillus filamentosus, Janibacter indicus and Brevibacterium casei isolated from the rhizosphere of Zygophyllum coccineum reduced the adverse effects of salinity in wheat plants. Studies on rhizobacteria present in tomato from the Al-Ahsa region have not 
been documented in the literature. Additionally, seeking bacterial strains with efficient plant growth-promoting traits and that have become adapted to the Al-Ahsa region's prevailing conditions is of unique importance compared to analyzing invaded strains. The present work, therefore, aimed to isolate and characterize these bacteria and assess their plant growth-promoting traits in three economically important crops, Eruca sativa, Lepidium sativum and Raphanus sativum of the region.

\section{Materials and Methods}

Collection of Solanum lycopersicum plants and bacterial isolation : S. lycopersicum plants were carefully uprooted along with rhizosphere from the Al-Ahsa region $\left(25^{\circ} 21^{11} 16.9^{\circ} \mathrm{N}\right.$, $\left.49^{\circ} 36^{1} 22.63 \mathrm{E}\right)$. One gram of rhizospheric soil was placed in a conical flask containing $99 \mathrm{ml}$ of $0.85 \% \mathrm{NaCl}$ solution. The flask was shaken thoroughly for 30 min under aseptic conditions. Then, a loopful of soil suspension was streaked onto King B media plates. Plates were incubated at $28^{\circ} \mathrm{C}$ for 3 days. Discrete single colonies were carefully picked and then purified by streaking onto King B media. Five bacterial isolates were obtained and preserved by subculturing on slants every 4 weeks, on a regular basis. Initial screening of isolates was carried out by assessing their plant growth promotion of seed germination of the three plants (data not shown). A focus was given to SLK10 due to its significant enhancing effects in plants.

\section{Phenotypic characterization of bacterial isolates}

Morphological characterization : SLK10 was characterized based on colony shape, length and Gram staining reaction (Table1) following the description as per Claus (1992).

Biochemical characterization using API20 kits : SLK10 was tested for its ability to consume 20 different metabolites contained in the API-20E test kit following the manufacturer's recommendations (Holmes et al., 1978). Incubation of inoculated strip was carried out at $30^{\circ} \mathrm{C}$, and the results were reported after $24 \mathrm{hr}$.

Growth of bacterial isolate under different concentrations of $\mathrm{NaCl}$ : SLK10 was assessed for its ability to grow under elevated levels of salt stress by streaking onto King B media supplemented with0, 1, 2, 3, 5 and $10 \% \mathrm{NaCl}$. After incubation period of $48 \mathrm{hr}$ at $30^{\circ} \mathrm{C}$ for, the plates were visually checked for bacterial growth. The experiments were performed in triplicate.

Testing for plant bio-stimulation traits : Phosphate solubilisation was checked following a qualitative method described earlier (Pikovskaya, 1948). SLK10 was streaked on Pikovskaya agar, which contains tricalcium phosphate $\left(\mathrm{Ca}_{3}\left(\mathrm{PO}_{4}\right)_{2}\right)$, a source of phosphate. Inoculated plates were incubated at $30^{\circ} \mathrm{C}$ for 5 days. Then, the plates were checked for the appearance of clear halo zones around the bacterial colonies, indicating positive results. The ability of SLK10 to produce the phytohormone IAA was assessed according to a previously described method (Gordon and Weber, 1951). Briefly, SLK10 was incubated with shaking (150 rpm) at $30{ }^{\circ} \mathrm{C}$ for $72 \mathrm{hr}$ after the isolated bacteria had been grown in nutrient broth (NB) with 0.0 , 0.1 and $0.2 \mathrm{mg} \mathrm{ml}^{-1}$ of tryptophan. Then, the cells were precipitated by centrifugation at $10000 \mathrm{rpm}$ for $10 \mathrm{~min}$. Two $\mathrm{ml}$ of Salkowski's reagent, which consisted of $150 \mathrm{ml}$ of concentrated sulfuric acid $\left(\mathrm{H}_{2} \mathrm{SO}_{4}\right), 250 \mathrm{ml}$ of distilled water and $7.5 \mathrm{ml}$ of half molar ferric chloride hexahydrate $\left(0.5 \mathrm{M} \mathrm{FeCl}_{3} \cdot 6 \mathrm{H}_{2} \mathrm{O}\right)$ were mixed with $1 \mathrm{ml}$ of the supernatant. Incubation of the tubes was carried out in darkness at $25^{\circ} \mathrm{Cfor} 25 \mathrm{~min}$. Then, the amount of IAA was estimated from a standard curve using a spectrophotometer at $\mathrm{OD}_{530} \mathrm{~nm}$.

The ability of SLK10 to produce catalase was checked by adding a drop of5\% hydrogen peroxide $\left(\mathrm{H}_{2} \mathrm{O}_{2}\right)$ to a smeared loopful of the growing isolate on a clean glass slide. Positive results were reported when gas bubbles were observed.

SLK10 was tested for its ability to fix atmospheric nitrogen by growing the strain in a nitrogen-free medium (Jensen's medium). After inoculation, plates were incubated at $30^{\circ} \mathrm{C}$ for 72 $h$, and the results were then recorded. The emergence of bacterial colonies was reported as a positive result.

Bacterial identification using 16S rRNA gene sequencing: Extraction of total genomic DNA from SLK10 was performed with a boiling method using an InstaGene Matrix (Bio-Rad, Hercules, CA) following the manufacturer's instructions. Briefly, a small section of growing bacterial colony was picked out using a sterilized toothpick, carefully suspended in $50 \mu$ lof $0.85 \% \mathrm{NaCl}$ and heated at $95^{\circ} \mathrm{C}$ for 5 min to release DNA from the lysed cells. After that, the cell debris was precipitated by centrifugation at $10000 \mathrm{rpm}$ for $10 \mathrm{~min}$. A $1 \mu$ laliquot of the supernatant served as template DNA for the subsequent PCR amplification steps. The universal primers 27F 5-AGA GTT TGA TCM TGG CTCAG-3 and 1492R 5-TACGGYTACCTTGTTACGACTT (Weisburg et al., 1991) were used to amplify the 16S rRNA gene for SLK10, using standard conditions (Khalifa and AIMalki, 2015). Sequencing of purified PCR product was conducted on an Applied Biosystems model 3730XL automated DNA sequencing system. The $16 \mathrm{~S}$ rRNA gene sequence of SLK10 was deposited in NCBI GenBank with accession number MW091474.The 16S rRNA gene similarity sequences were compared to those in the GenBank database using global alignment algorithm at the EzTaxon server (http://www.eztaxon.org) (Kim et al., 2012). Phylogenetic trees were constructed with the MEGA7.0 program, following neighbour-joining method based on the Tamura-Nei model (Tamura and Nei, 1993).

Effects of bacterial isolates on various plant species : Seeds of Eruca sativa Mill (rocket), Lepidium sativum L. (garden cress) and Raphanus sativus (radish) were purchased from local markets. Healthy seeds were surface-sterilized with $70 \%$ ethanol 
for $10 \mathrm{sec}$, then with $\mathrm{H}_{2} \mathrm{O}_{2}(3 \%)$ for 3 min and finally washed with sterilized distilled water five times. Inocula of strain SLK10 were prepared as outlined previously (Khalifa et al., 2019). Plant seeds were inoculated with a $3 \times 10^{6} \mathrm{CFU} \mathrm{ml}{ }^{-1}$ bacterial suspension of SLK10 for $24 \mathrm{hr}$ under aseptic conditions. Three concentrations of $\mathrm{NaCl}(1000,2000$ and $3000 \mathrm{mM})$ were applied to each inoculated seed. Seeds were grown in plastic pots filled with $0.5 \mathrm{~kg}$ sterilized soil in a growth chamber at $22^{\circ} \mathrm{C}$, relative humidity of $50 \%$ and a photoperiod of $14 \mathrm{~h}$. Controls without added salt or bacteria were used. Experiments were performed in triplicate. After three weeks, root and shoot height, and the number of leaves were measured and compared with non-treated seeds.

Statistical analyses : Data were statistically analyzed using CoStat version 6.303 1998-2004 CoHort software 798 Lighthouse Ave PMP 320, Monterey, CA, 93940, USA. Analysis of variance (ANOVA) and Duncan's Multiple Range Test at $P \leq 0.05$ were performed to compare the results.

\section{Results and Discussion}

The current study aimed to isolate and characterize bacterial isolates inhabiting the rhizosphere of Solanum lycopersicum in the Al-Ahsa region and estimate the biostimulation effects on three different plant species growing under salt stress.

Fivebacterial isolates were isolated from the rhizospheric soils of Solanum lycopersicum growing in Al-Ahsa. SLK10 was selected for further investigation based on the significance results obtained upon inoculation of three plant species under the three salt stress levels tested. SLK10 was further characterized based on its morphological, biochemical, genotypic and phylogenetic characteristics (Table1). SLK10 formed a circular, non-pigmented and raised colony with an entire margin. The size of the colony was $2-5 \mathrm{~mm}$, on nutrient agar after $24 \mathrm{hr}$ at $30^{\circ} \mathrm{C}$. The cells were rod shaped and Gram negative. SLK10 was characterized using the API 20E biochemical identification system, and the results obtained are displayed in Table 1. SLK10 was diverse with respect to the biochemical characteristics revealed by API20 E. SLK10 consumed ortho-nitrophenyl- $\beta$-galactoside (ONPG), arginine, citrate and tryptophan. Among the carbohydrates tested, SLK10 consumed inositol, sorbitol and glucose. Furthermore, SLK10 exhibited no ability to ferment/oxidize mannitol, rhamnose, sucrose, melibiose, amygdalin and arabinose as the only carbon source. SLK10 was diverse with respect to the API 20E results. These findings are in accordance with those obtained for Pseudomonas sp. isolated from sugar cane and rice fields (Yasmin et al., 2020). This finding indicates the metabolic versatility of SLK10 strain. Interestingly, SLK10 could enhance plant growth via ammonia production via arginine dihydrolase or acetoin production. Ammonia production is a source of nitrogen in plants and participates in biochemical reactions for amino acid synthesis. Therefore, ammonia- producing PGPR promotes plant growth and biomass production. In this study, five bacterial isolates produced ammonia. This finding coincides with the results of Pal et al. (2019), who found that ammonia was produced by Pseudomonas. Acetoin is an elicitor of induced systemic resistance of plants against pathogenic agents.

The bacterial isolates in this study were able to utilize a range of substrates in the API $20 \mathrm{E}$ tests is important in light of the fact that root secretions contain a wide range of useful compounds that can attract microorganisms and change the chemical and physical properties of soil. Such secretions vary depending on plant development and plant type, producing different microbial communities that vary in diversity (Bishnoi, 2015). The morphological and biochemical characteristics of the strain SLK10 are in accordance with those reported for $P$. monteilii (Elomari et al., 1997), confirming the identity of bacterial strain at phenotypic level.

As can be seen in Table 1, SLK10 grew well at 0,1,2 and $3 \% \mathrm{NaCl}$. No growth was observed at $5 \% \mathrm{NaCl}$. This finding indicates that SLK10 is a halotolerant plant growth-promoting bacterial strain that can provide a selective advantage for plants growing in saline conditions.

SLK10 was able to grow on Jensen's medium, which contains no added nitrogen source and is designed for detecting $\mathrm{N}_{2}$-fixing bacteria. This finding highlights that this isolate can fix nitrogen (Table1), an ability that is attributed to the presence of the nitrogenase enzyme. These results corroborates with the study of Verma et al. (2016), who documented nitrogen fixation in Pseudomonas spp. Nitrogen-fixing PGPR plays a key role in fulfilling plants' requirement for the essential macronutrient nitrogen, thereby increasing soil fertility and plant productivity.

SLK10 formed a clear zone around the colonies grown on Pikovskaya's agar, showing ability for phosphate solubility (Table1). Phosphorus is one of the most important and limiting macronutrients for plant growth. Even though its level in soil is high, phosphorus is unavailable to plants. Therefore, bacteria can increase the availability of phosphorus and promote plant growth (Khalifa et al., 2016). SLKI was able to solubilise phosphorus. This observation could be explained by the potential ability of both strains to secrete phosphatases and organic acids. Therefore, plants can easily obtain this essential nutrient. These findings are in accordance with earlier studies of phosphate-solubilizing bacteria P. putida (Pal et al., 2019), Pseudomonas sp. from Lotus creticus and $P$. aeruginosa from Capsicum annuum L. (Achkouk et al., 2019). SLK10 was characterized by phosphate solubility as a PGP feature, which could improve plant growth via its role in increasing phosphorus bioavailability for plants.

SLK10 produced the phytohormone-IAA-when grown on 0.1 and $0.2 \mu \mathrm{g} \mathrm{ml}^{-1}$ tryptophan. The amount of IAA produced by 
Table 1: Characterization of strain SLK10

Morphological characteristics of strain SLK10

\begin{tabular}{ll}
\hline Colony shape & Circular \\
Pigmentation & Non-pigmented \\
Margin & Entire \\
Gram reaction & Negative \\
Cell shape & Rods
\end{tabular}

Biochemical characterization using API 20E

\begin{tabular}{|c|c|c|c|}
\hline Number & Substrate & Enzyme involved/reaction tested & Result \\
\hline 1 & ONPG * & Beta-galactosidase & + \\
\hline 2 & Arginine & Arginine dihydrolase & + \\
\hline 3 & Lysine & Lysine decarboxylase & " \\
\hline 4 & Ornithine & Ornithine decarboxylase & " \\
\hline 5 & Citrate & Citrate utilization & + \\
\hline 6 & Na thiosulfate & $\mathrm{H}_{2} \mathrm{~S}$ production & + \\
\hline 7 & Urea & Urea hydrolysis & " \\
\hline 8 & Tryptophan & Deaminase & + \\
\hline 9 & Tryptophan & Indole production & + \\
\hline 10 & Na pyruvate & Acetoin production & + \\
\hline 11 & Charcoal gelatin & Gelatinase & - \\
\hline 12 & Glucose & Fermentation/oxidation & + \\
\hline 13 & Mannitol & & - \\
\hline 14 & Inositol & & + \\
\hline 15 & Sorbitol & & + \\
\hline 16 & Rhamnose & & " \\
\hline 17 & Sucrose & & - \\
\hline 18 & Melibiose & & - \\
\hline 19 & Amygdalin & & - \\
\hline 20 & Arabinose & & - \\
\hline \multicolumn{4}{|c|}{ Plant growth-promoting characteristics of strain SLK10 } \\
\hline \multicolumn{2}{|c|}{ Phosphate solubility } & \multicolumn{2}{|l|}{+} \\
\hline \multicolumn{2}{|c|}{ 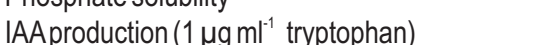 } & \multicolumn{2}{|l|}{$0.33 \pm 0.014 \mu \mathrm{g} \mathrm{ml}^{-1}$} \\
\hline \multicolumn{2}{|c|}{ IAA production ( $2 \mu \mathrm{g} \mathrm{ml}^{-1}$ tryptophan) } & \multicolumn{2}{|l|}{$0.80 \pm 0.019 \mu \mathrm{g} \mathrm{ml}^{-1}$} \\
\hline \multicolumn{2}{|c|}{ Nitrogen fixation } & \multicolumn{2}{|l|}{+} \\
\hline \multicolumn{2}{|l|}{ Catalase } & \multicolumn{2}{|l|}{+} \\
\hline \multicolumn{2}{|c|}{ Growth at $0,1,2$ and $3 \% \mathrm{NaCl}$} & \multicolumn{2}{|l|}{+} \\
\hline \multicolumn{2}{|c|}{ Growth at $5 \% \mathrm{NaCl}$} & \multicolumn{2}{|l|}{-} \\
\hline \multicolumn{4}{|c|}{ Genotypic characteristics of strain SLK10 } \\
\hline \multirow{2}{*}{\multicolumn{2}{|c|}{$\begin{array}{l}\text { Accession number of the } 16 \text { s rRNAgene } \\
\text { Sequence homology to } P \text {. monteilii }\end{array}$}} & \multicolumn{2}{|l|}{ MW091474 } \\
\hline & & $98.66 \%$ & \\
\hline
\end{tabular}

+ : means positive result and -: means negative result, ${ }^{*}$ : ortho-nitrophenyl- $\beta$-galactoside (ONPG).

SLK10 was0.33 $\pm 0.014 \mu \mathrm{g} \mathrm{ml}^{-1}$ when grown on $1 \mu \mathrm{g} \mathrm{ml}{ }^{-1}$ tryptophan and increased to $0.80 \pm 0.019 \mu \mathrm{g} \mathrm{m}^{-1}$ when 0.1 and 0.2 $\mu \mathrm{g} \mathrm{m}^{-1}$ tryptophan was added to the growth medium (Table 1). No IAA was detected when SLK10 was grown with no added tryptophan. The production of phytohormones is another trait that promotes plant growth. IAA is a growth regulator that promotes seed germination and plant-cell division and controls vegetative growth. However, only a relatively small concentration of IAA is required for plant root development. SLK10produced IAA (0.80 \pm
$0.019 \mu \mathrm{g} \mathrm{ml}^{-1}$ ). Comparable concentrations of IAA were reported for Pseudomonas sp. (Sharma et al., 2018). These findings indicate that IAA production is an important feature of the plant growth-promoting bacteria, and SLK10 was no exception in this regard.

SLK10 formed an immediate effervescence and formation of gas bubbles upon addition of hydrogen peroxide to the growing culture, indicating that this isolate was catalase 


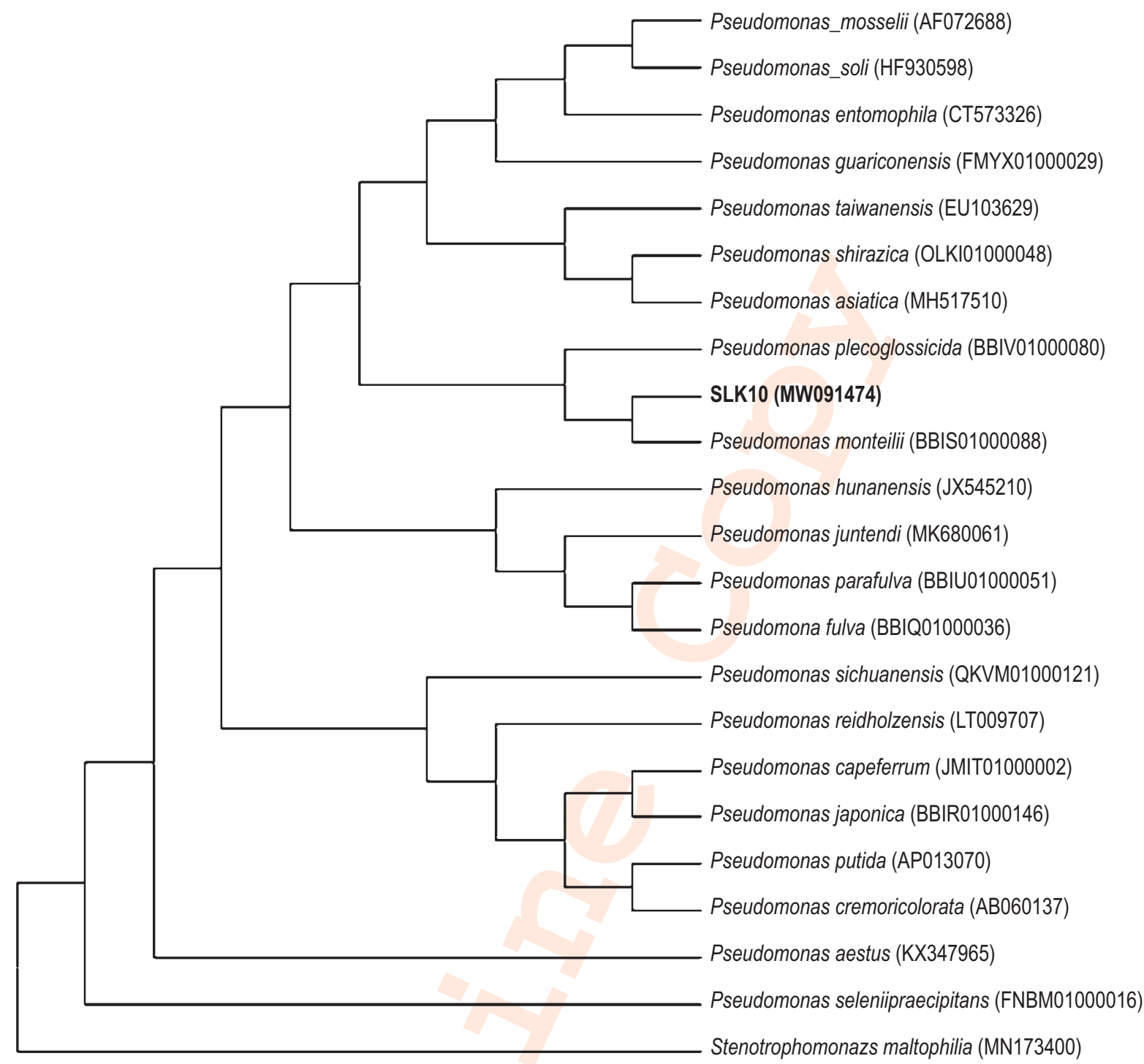

Fig. 1 : Phylogenetic tree showing the relationships among the strain SLK10 with our and other related defined species, as inferred using the neighbourjoining method. Analyses were conducted in MEGA7.0.

positive (Table1). The enzyme catalase plays an important role in protecting organisms from toxic free radicals, which are particularly formed under environmental stresses. Therefore, bacterial catalase production can promote plant growth via this indirect mechanism. The production of catalase enzyme by the bacterial isolates is in accordance with previous studies on Pseudomonas sp. (Sharma et al., 2018). These observations highlights that catalase is an important enzyme in promotion of plant growth.

The 16S rRNA gene of the strain SLK10 was sequenced, and the high-quality sequences were deposited in the NCBI database under the accession number SLK10 (MW091474) (Table 1). A comparative sequence analysis of 16S rRNA gene revealed that the SLK10 strain belonged to $P$. monteilii (accession number BBIS01000088), exhibiting $98.66 \%$ sequence homology. A neighbour-joining phylogenetic tree constructed using $16 \mathrm{~S}$ rRNA gene sequences of closely related recognized species showed the taxonomic affiliation of the strain SLK10 (Fig. 1). SLK10 (accession number MW091474) was clearly clustered with the members of Pseudomonas spp. and formed a monophyletic subgroup with P. monteilii (BBIS01000088) (Fig. 1). This observation provides robustness for the taxonomic affiliation of the strain SLK10 and confirming the efficiency of 16S rRNA gene sequences as a powerful fingerprint tool for bacterial identification. The taxonomic and phylogenetic results are consistent with the findings of morphological characterization of SKL10 (i.e., Gram negative with entire, circular and convex 


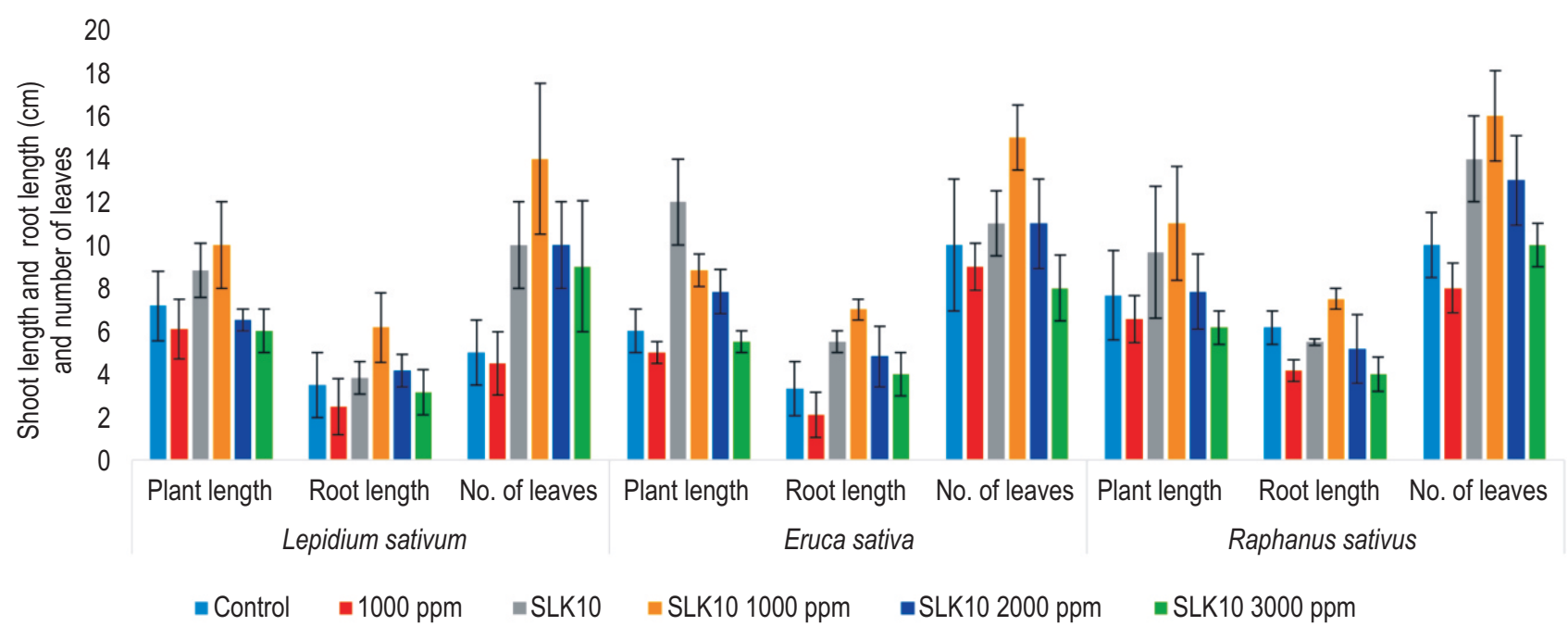

Fig. 2 : Results of growth parameters of Eruca sativa Mill (rocket), Lepidium sativum L. (garden cress) and Raphanus sativus L. (radish) growing under different levels of salinity stress when inculated with SLK10.

colonies). In addition, the morphological characteristics of SKL10 were consistent with those of Elomari et al. (1997) and Sandhya et al. (2009) regarding Pseudomonas sp. features. Consistency in morphological, taxonomic and phylogenetic characterizations provide robustness in bacterial designation of SKL10 as $P$. monteilii.

SLK10 significantly promoted the length of primary root, stem and number of leaves in $E$. sativa, L. sativum and $R$. sativus growing under $1000 \mathrm{ppm}$ salt stress. The number of leaves in $L$. sativum and $R$. sativus growing under $2000 \mathrm{ppm} \mathrm{NaCl}$ salt stress was substantially enhanced by SLK10 (Fig. 2). However, no significant enhancement was observed in the growth parameters of three plant species growing under $3000 \mathrm{ppm} \mathrm{NaCl}$ salt stress when inoculated with SLK10. Under a salinity level of $1000 \mathrm{ppm}$, SLK10 significantly increased the shoot length by $39.5 \%, 47.2 \%$ and $43.7 \%$ in L. sativum, E. sativa and $R$. sativus, respectively (Figs. 2 and 3). A similar significant improvement in root length by $76.0 \%, 110.2 \%$ and $21.75 \%$ was noted in L. sativum, E. sativa and $R$. sativus, respectively (Table 2).SLK10 significantly enhanced the number of leaves $100 \%$, in L. sativum and from $30 \%$ in R. sativus (Figs. 2 and 3 ).

The PGP effects of SLK10 bacterial strain was explored, showing that it has multiple PGP traits for three plant species growing under salt stress. These features make the isolates detected here potentially beneficial for biofertilizers. In previous research, bacterial strains have been used as biofertilizers in various crops, such as green bean, corn, wheat, cotton, soybean and forage ones (Bishnoi, 2015).

The findings that Pseudomonas sp. (SKL10) promoted growth root length of $L$. sativum, $E$. sativa and $R$. sativus are in agreement with the observations of Kumar et al. (2016) on beans inoculated with Pseudomonas sp. Similarly, Pseudomonas sp. increased the height and dry weight of tomato plant roots (Ricci et al., 2019). Such positive improving effects could be attributed to the multiple plant growth features of our strain, SKL10.

The stimulation of root growth parameters of three plant species described here could be attributed to multiple plant growth-enhancing traits of our bacterial isolates. The results also confirm that multifunctional plant growth-promoting features are common among rhizobacteria.

Alleviation of harmful effects of salinity stress of $L$. sativum, $E$. sativaand $R$. sativus by inoculating with SLK10 indicates its potential plant growth-promoting traits. These observations are in agreement with those obtained by OrozcoMosqueda et al. (2019), who reported a protective role of Pseudomonas sp. UW4 against the negative effects of salinity stress in tomato plants (Orozco-Mosqueda et al., 2019). A possible explanation of this role is a synergistic action of trehalose, a non-reducing disaccharide, and ACC deaminase. Whether or not ACC deaminase and trehalose have same mechanism in our study requires further investigation. Additionally, inoculation with PGPR has been reported to upregulate the salt-responsive genes. Further, Pseudomonas spp. have been documented to alleviate salinity stresses in various plant species, including tomato (Orozco-Mosqueda et al., 2019) and soybean (Yasmin et al., 2020) via reduction of toxic ion uptake, induction of systemic resistance and production of plant hormones and antioxidants.

The genome of $P$. monteilii CY06 has been sequenced (Ma et al., 2019). This information provides substantial promise for in-depth studies to decipher the mechanism via which this bacterium alleviates the negative effects of salinity. Focus should 
be given to the up- and down-regulated genes that are encoding plant growth promoting traits such as IAA production.

In conclusion, P. monteilii SLK10 was isolated from the herbaceous plant S. Iycopersicum and showed multiple plant growth-promoting features and significantly improved the growth parameters of Lepidium sativum, Eruca sativa and Raphanus sativus under salt stress. These findings suggest that this strain can be developed as a promising green biofertilizer in the future.

\section{Acknowledgments}

The authors extend their gratitude to the Deanship of Scientific Research (DSR), King Faisal University, Saudi Arabia, for providing funding for the current work (DSR, project No. 180085).

\section{Add-on Information}

Authors' contribution: M.F. Aldayel: Obtained the Fund, conducted experimental work, revise the manuscript, A. Khalifa: Conducted experimental work, Wrote the manuscript.

Research content: The research content of manuscript is original and has not been published elsewhere.

\section{Ethical approval: Not applicable}

Conflict of interest: The authors declare that there is no conflict of interest.

\section{Data from other sources: Not applicable}

Consent to publish: All authors agree to publish the paper in Journal of Environmental Biology.

\section{References}

Abbamondi, G.R., G. Tommonaro, N. Weyens, S. Thijs, W. Sillen, P. Gkorezis and J. Vangronsveld: Plant growth-promoting effects of rhizospheric and endophytic bacteria associated with different tomato cultivars and new tomato hybrids. Chem. Biol. Technol. Agric., 3, 1-10 (2016).

Achkouk, I., S. Aarab, A. Laglaoui, M. Bakkali and A. Arakrak: Isolation and screening of inorganic phosphate solubilizing Pseudomonas strains from the Lotus creticus rhizosphere soil from the northwest of Morocco. Phyto-Microbio. Stress Regulat., 99-111 (2020).

AlAli, H. A., A. Khalifa and M. Al-Malki: Plant growth-promoting rhizobacteria from Ocimum basilicum improve growth of Phaseolus vulgaris and Abelmoschus esculentus. S. Afr. J. Bot., 139, 200-209 (2021).

Bishnoi, U.: PGPR Interaction: An eco friendly approach promoting the sustainable agriculture system. Adv. Bot. Res., 75, 81-113 (2015).

Chinnaswamy, A., T. Coba de la Peña, A. Stoll, D. de la Peña Rojo, J. Bravo, A. Rincón and J.J. Pueyo: A nodule endophytic Bacillus megaterium strain isolated from Medicago polymorpha enhances growth, promotes nodulation by Ensifer medicae and alleviates salt stress in alfalfa plants. Ann. Appl. Biol., 172, 295-308 (2018).

Claus, D.: A Standardized Gram Staining Procedure. World J. Microbiol. Biotechnol., 8, 451-452 (1992).

Elomari, M., L. Coroler, S. Verhille, D. Izard and H. Leclerc: Pseudomonas monteilii sp. nov., isolated from clinical specimens. Int. J. Syst. Evol. Microbiol., 47, 846-852 (1997).

Gamez, R.M., S. Ramirez, M. Montes and M. Cardinale: Complementary dynamics of banana root colonization by the plant growthpromoting rhizobacteria Bacillus amyloliquefaciens Bs006 and Pseudomonas palleroniana Ps006 at spatial and temporal scales. Microb. Ecol., 80, 656-668 (2020).

Gordon, S.A. and R.P. Weber: Colorimetric estimation of indoleacetic acid. Plant Physiol., 26, 192 (1951).

Holmes, B., W.R. Willcox and S.P. Lapage: Identification of Enterobacteriaceae by the API 20E system. J. Clin. Pathol., 31, 2230 (1978).

Khalifa, A.Y. and M.A. Almalki: Isolation and characterization of an endophytic bacterium, Bacillus megaterium BMN1, associated with root nodules of Medicago sativa L. growing in Al-Ahsaa Region, Saudi Arabia. Ann. Microbiol., 65, 1017-1026 (2015).

Khalifa, A.Y. and M. Almalki: Polyphasic characterization of Delftia acidovorans ESM-1, a facultative methylotrophic bacterium isolated from rhizosphere of Eruca sativa. Saudi J. Biol. Sci., 26, 1262-1267 (2019)

Khalifa, A.Y., A.M. Alsyeeh, M.A. Almalki and F.A. Saleh: Characterization of the plant growth promoting bacterium, Enterobacter cloacae MSR1, isolated from roots of non-nodulating Medicago sativa. Saudi J. Biol. Sci., 23, 79-86 (2016).

Khalifa, A.Y., A. Metwally, R.B. Ammar and F.A. Farghaly: ACC Deaminase-containing rhizobacteria from rhizosphere of Zygophyllum coccineum alleviate salt stress impact on wheat (Triticum aestivum L.). Sci. J. King Faisal Univ., 21, 89-101 (2020).

Kim, O.S., Y.J. Cho, K. Lee, S.H. Yoon, M. Kim, H. Na and J. Chun: Introducing EzTaxon-e: A prokaryotic 16S rRNA gene sequence database with phylotypes that represent uncultured species. Int. J. Syst. Evol. Microbiol., 62, 716-721 (2012).

Kumar, P., R.C. Dubey, D.K. Maheshwari, Y.H. Park and V.K. Bajpai: Isolation of plant growth-promoting Pseudomonas sp. ppr8 from the rhizosphere of Phaseolus vulgaris I. Arch. Biol. Sci., 68, 363-374 (2016).

Liao, C.Y. and D.C. Bassham: Combating stress: The interplay between hormone signaling and autophagy in plants. J. Exp. Bot., 71, 1723-1733(2020).

Ma, Q., Y. Cai and Z. He: Complete genome sequence of a novel aerobic denitrifying strain Pseudomonas monteilii CY06. Mar. Genomics, 47, 100661 (2019)

Orozco-Mosqueda, M., J. Duan, M. DiBernardo, E. Zetter, J. CamposGarcía, B.R. Glick and G. Santoyo: The production of ACC deaminase and trehalose by the plant growth promoting bacterium Pseudomonas sp. UW4 synergistically protect tomato plants against salt stress. Front. Microbiol., 10, 1392 (2019).

Ossowicki, A., S. Jafra and P. Garbeva: The antimicrobial volatile power of the rhizospheric isolate Pseudomonas donghuensis P482. PloS ONE, 12, e0174362 (2017).

Pal, A.K. and C. Sengupta: Isolation of cadmium and lead tolerant plant growth promoting rhizobacteria: Lysinibacillus varians and Pseudomonas putida from Indian agricultural soil. Soil. Sedim. Contam., 28, 601-629 (2019).

Pandya, N.D. and P.V. Desai: Screening and characterization of GA3 
producing Pseudomonas monteilii and its impact on plant growth promotion. Int. J. Curr. Microbiol. Appl. Sci, 3, 110-115 (2014).

Pikovskaya, R.: Mobilization of phosphorus in soil in connection with vital activity of some microbial species. Mikrobiologiya, 17, 362-370 (1948).

Qessaoui, R., R. Bouharroud, J.N. Furze, M. El Aalaoui, H. Akroud, A. Amarraque and B. Chebli: Applications of new rhizobacteria Pseudomonas isolates in agroecology via fundamental processes complementing plant growth. Sci. Rep., 9, 1-10 (2019).

Raza, A., M.B. Hafeez, N. Zahra, K. Shaukat, S. Umbreen, J. Tabassum and M. Hasanuzzaman: The plant family Brassicaceae: Introduction, biology, and importance. In: The Plant Family Brassicaceae . Springer, Singapore, pp. 1-43 (2020).

Ricci, E., T. Schwinghamer, D. Fan, D.L. Smith and V. Gravel: Growth promotion of greenhouse tomatoes with Pseudomonas sp. and Bacillus sp. biofilms and planktonic cells. Appl. Soil Ecol., 138, 61-68 (2019).

Sandhya, V., M. Grover, G. Reddy and B. Venkateswarlu: Alleviation of drought stress effects in sunflower seedlings by the exopolysaccharides producing Pseudomonas putida strain GAPP45. Biol. Fertil. Soils, 46, 17-26 (2009).

Shameer, S. and T. Prasad: Plant growth promoting rhizobacteria for sustainable agricultural practices with special reference to biotic and abiotic stresses. Plant Growth Regul., 84, 603-615 (2018).

Sharma, S., A. Sharma and M. Kaur: Extraction and evaluation of gibberellic acid from Pseudomonas spp. plant growth promoting rhizobacteria. J. Pharmacogn. Phytochem., 7, 2790-2795 (2018).

Singh, A.: Soil salinization management for sustainable development: A review. J. Environ. Manag., 277, 111383 (2021).

Tamura, K. and M. Nei: Estimation of the number of nucleotide substitutions in the control region of mitochondrial DNA in humans and chimpanzees. Mol. Biol. Evol., 10, 512-526 (1993).

Verma, P., A.N. Yadav, K.S. Khannam, S. Kumar, A.K. Saxena and A. Suman: Molecular diversity and multifarious plant growth promoting attributes of Bacilli associated with wheat (Triticum aestivum $\mathrm{L}$.) rhizosphere from six diverse agro ecological zones of India. J. Basic Microbiol., 56, 44-58 (2016).

Weisburg, W.G., S.M. Barns, D.A. Pelletier and D.J. Lane: 16S Ribosomal DNA amplification for phylogenetic study. J. Bacteriol., 173, 697-703 (1991).

Yasmin, H., S. Naeem, M. Bakhtawar, Z. Jabeen, A. Nosheen, R. Naz and M.N. Hassan: Halotolerant rhizobacteria Pseudomonas pseudoalcaligenes and Bacillus subtilis mediate systemic tolerance in hydroponically grown soybean (Glycine max L.) against salinity stress. PLOS ONE, 15, e0231348 (2020). 